\title{
A survey on experimental elicitation of creativity in economics
}

\author{
Giuseppe Attanasi ${ }^{* 1,3}$, Michela Chessa ${ }^{\dagger 1}$, Sara Gil Gallen ${ }^{\ddagger 2}$, and Patrick Llerena ${ }^{\S 3}$ \\ ${ }^{1}$ Université Côte d'Azur, CNRS, GREDEG, France \\ ${ }^{2}$ Università degli studi di Bari "Aldo Moro", Italy \\ ${ }^{3}$ BETA, University of Strasbourg, France
}

\begin{abstract}
The interplay between individual creative ability and the way to enhance it - through monetary and nonmonetary incentives - is an issue with tremendous potential for economic analysis. In this survey, we dwell into the issue by focusing on the methodological advantages of economic experiments. We provide a review of the literature in experimental economics on creativity, identifying six main directions of analysis. Namely, the impact on creativity of: (1) low vs high monetary incentives, (2) the interplay of monetary incentives and tasks, (3) within-group competition, (4) within-group cooperation, (5) cultural factors, and (6) non-monetary social incentives. In the spirit of a "meta-study," we classify the works in our review not only according to the aforementioned research questions, but also disentangling by the type of creative task that the experimental subjects face, the way in which creativity is assessed, and other key features of standard experimental procedures in economics. This multidimensional comparison allows us to conclude that the current lack of robust findings on the determinants of creativity in economics might be due to the absence of comparable experimental studies under the same experimental conditions. We conduct our analysis without neglecting the psychological roots of creativity research and their way through management, underlying how both disciplines have heavily outlined the work of experimental economists in the topic of creativity.
\end{abstract}

JEL codes: C91, C92, D91, O31

Keywords: Creativity, Experimental Economics, Social Psychology, Incentives, Intrinsic Motivation

Acknowledgements: We are grateful to participants at the workshop "La créativité: Les apports de l'histoire des idées et des sciences sociales" at Université Côte d'Azur, Nice for useful comments. The research leading to these results has received funding from the French Agence Nationale de la Recherche (ANR) under grant ANR-18-CE26-0018-01 (project GRICRIS) and from the Chair in Management of Creativity at the Université de Strasbourg.

\footnotetext{
*Corresponding author: giuseppe.attanasi@univ-cotedazur.fr

${ }^{\dagger}$ michela.chessa@univ-cotedazur.fr

${ }^{\ddagger}$ saragilgallen@gmail.com

§ pllerena@unistra.fr
} 


\section{Introduction}

Creativity contributes to life quality and progress in a variety of dimensions, ranging from dance, art, music and writing, to economics, medicine, mathematics, etc. The modern notion of creativity, reported by [Mednick, 1962, p.220-221], refers to Henri Poincaré who, in 1908, stated that "to create consists of making new combinations of associative elements which are useful. [...] Among chosen combinations the most fertile will often be those formed of elements drawn from domains which are far apart." During the last century, creativity has been studied from different perspectives by scientists working in social and cognitive sciences, e.g., anthropology, sociology, psychology, management and economics. Given the interdisciplinarity of research in creativity, different definitions of creativity have been provided along the years [see Ochse, 1990]. However, the overwhelming majority of them agrees on indicating creativity as the ability to do new things which have some utility.

Built on this conceptualization, psychological tests have represented, for over half a century, the standardbearer for researchers to detect creative ability and even creative personality, as a psychological assessment of human potential and performance. The research interest to identify creative persons might lead to think that creativity is simply a special quality of unusual people. This is the impression that creativity research could provide at the beginning of the 70s. That was also the time when Teresa Amabile, the reference author in the studies about creativity in psychology, started exploring the existing literature, out of a long-standing curiosity about the subject. Citing her words, the predominant impression the literature was providing at the time being was that "creativity is a quality of the person; most people lack that quality; people who possess the quality-geniuses-are different from everyone else, in talent and personality; we must identify, nurture, appreciate, and protect the creatives among us-but, aside from that, there isn't much we can do" [Amabile and Pillemer, 2012, p.3].

Since then, the researches of Amabile [1982a,b, 1983, 1985] and Simonton [1975, 1980] and the interaction between the two authors in the so-called social psychology of creativity aimed at contradicting the standing simplified vision of the problem, showing, both at a micro and at a macro level respectively, how various social, cultural and political factors affect creative success, in addition to the obvious ability and personality traits. Amabile's reflection (with the important influence of Simonton's work) has resulted in what she called componential theory of creativity [Amabile, 1983], where the social environment is identified as the one external component which influences creativity, next to the more known individual factors. As resumed by [Attanasi et al., 2019a, p.2] in a recent work on individual vs. group creativity in economics, 
since then researchers agreed on the fact that "creativity resides in the personal capacities of individuals, in the form of native talent and/or acquired know-how, but it is also embedded in concrete organizational contexts that shape its motions and objectives in many different ways."

As a result of this awareness, the most recent and widespread view of academics is that this relation between the individual and his/her environment is in fact bi-directional. Not only social, economical and cultural interactions influence the salience of subject's creativity, but, on the other side, releasing and enhancing creative abilities is possible, and this can in turn produce significant social and economical consequences. Then, the task of studying how to stimulate creativity turned out to be extremely valuable and profitable, and the one which was simply a topic within psychology, sociology, and cognitive science, became a subject of interest to other disciplines, such as management and economics. We might resume the time-line of the research on the interplay between intra-individual components and the social environment in the production of creative ideas as follows. As explained above, the first studies in psychology on this issue date back to the 80s [see Amabile, 1982a, and follow-up papers]. The management literature started to put interest on the subject in the 90 s, due to its historical focus on the management of innovation [see the survey of Burger-Helmchen et al., 2016]. In fact, while innovation and creativity are two different but linked concepts, the former greatly depends on the human ability to create. Creativity is a human act that involves the generation of novel and useful ideas, and innovation entails the implementation of these ideas into new products and processes. Therefore, studying how to incentivize the production of novel and useful ideas was meant to find ways to boost innovation. These management studies on the links between creativity and innovation represented the bridge between research in psychology and the one in economics. The first economic works analyzing the individual and social determinants of creativity only appeared with the new millennium. Nowadays, this topic is investigated in all the three mentioned fields. Despite different formalizations of the aforementioned research question in the three different fields, they all share at least one common methodology, i.e., the use of laboratory experiments. In fact, given the need of collecting empirical evidence, and due to the scarcity of field-data unambiguously linking incentives and creativity, the natural way to perform clean tests has turned to the implementation of controlled laboratory experiments. These are characterized by a controlled setting where the impact of each idiosyncratic feature and of different environmental incentives to creativity can be studied in isolation through treatment manipulations. This allows to state the impact of a specific incentive to creativity generation in a setting that is not contaminated with other real-life motivations.

An example may help clarifying the importance of the experimental methodology. In 2000 the Mil- 
lennium Prize Problems were released. They promised a US\$1 million prize to the solver of each of the historical mathematical "enigmas," such as the Riemann hypothesis or the Poincaré conjecture. The latter, in particular, is the only one which has been solved, only 3 years later. Surprisingly, Grigori Perelman, "the winner," declined the monetary prize. Some natural questions arise. Is he a genius? Probably yes. Did he solve the problem because of the monetary prize? Surely not. Did the social incentive of becoming the first one to reach such a goal play a role? Maybe. In our view, this is an example of how non-monetary incentives to creativity, e.g., the intrinsic motivation to find a creative solution to a complex problem, or the social motivation to be the first one to solve it, play a role. And how difficult it is to identify them in real-life situations without including confounding factors.

More in general, the interplay between individual creative ability and the way to enhance it - through monetary and non-monetary incentives - is an issue with tremendous potential for economic analysis. In this survey, we dwell into the aforementioned issue by focusing on the methodological advantages of economic experiments. We place ourselves in between the pretty few works that have already been produced and the many more to come, with the ambition of tidying up things for future researchers in the field. Our survey is meant to offer an overview about the multidisciplinary background, the existing literature and the future lines of experimental investigation of incentives to creativity.

More precisely, we provide a review identifying six main directions of analysis that embed most experimental research on creativity in economics. More precisely, the impact on creativity of: (1) low vs high monetary incentives, (2) the interplay of monetary incentives and tasks, (3) within-group competition, (4) within-group cooperation, (5) cultural factors, and (6) non-monetary social incentives.

Most importantly, we conduct our analysis without neglecting the psychological tradition and roots of creativity research, that have heavily outlined the work of economists. For this reason, in Section 2, we start our survey by listing some "pillars" concepts in social and psychological research in creativity. In the last part of this section, we open a bracket on how these concepts went through management research, which represents, in the topic of creativity, the bridge between research in psychology and the one in economics. In Section 3, we zoom on the research on creativity in experimental economics: given the newborn interest in the subject of this discipline, we organize the many different definitions, terminologies and methods. In Section 4 , we finally present our review of works in experimental economics on the topic of creativity, listed according to the aforementioned six directions. Section 5 concludes. 


\section{The pillars: psychological research in creativity and its way along to man- agement}

\subsection{Personality traits}

The first and most controversial pillar of research on creativity in psychology is about how personality traits affect creative performances. Since the earliest studies, researchers have focused their attention on how some people are consistently able to come up with novel and adaptive solutions and most others are not. This awareness is at the basis of the longstanding idea that creativity is in the hands of few ones, the previously mentioned geniuses, who naturally own the required qualities for accomplishing out-of-the-box targets. For this reason, the landmark works in the field involved deep studies of few widely recognized creators, in different disciplines such as architecture, mathematics, and writing, and the comparison with the profiles of some less-successful peers [see Barron, 1961, Mackinnon, 1965]. The ambition was to define which aspects of background or personality traits were co-varying with creative performances, while making a comparison between the more and the less creative individuals.

The creative measurement tests that were proposed just after these studies had the clear goal of putting these insights into practical use, providing researchers of a validated instruments to detect creative ability and personality (see Section 2.2). Since Amabile's revolution, most recent works have set aside this idea of personal traits as the only engines of creativity, while still recognizing their essential, complementary role in the process. For this reason, the research on how individual characteristics are related to creativity is still a flourishing topic, which has taken many different angles of analysis. In the impossibility of presenting an exhaustive review, we discuss few of them.

A large part of the literature has proposed and investigated a relationship between risk attitude and creativity. Early studies such as the one of Merrifield et al. [1961] have shown a significant correlation between some measures of participants' creativity and their level of risk taking. In the same direction, we may report similar results in Glover and Sautter [1976] and Glover [1977], who found that high-risk takers were significantly more flexible and original than low-risk takers in the creative task. The work of Erbas and Bas (2015) is instead upstream, as the authors did not find any significant correlation. In general, there is a lack of consensus regarding the relationship between risk taking and creativity, which can be attributed to the dependence of the former on the specific elicitation method. ${ }^{1}$ We refer to a recent survey by Tyagi et al.

\footnotetext{
${ }^{1}$ See Crosetto and Filippin [2016] and Attanasi et al. [2018] as for robust experimental evidence that subjects' estimated risk attitude parameters vary greatly across elicitation tasks.
} 
[2017] for a systematic exploration of this association in the psychological literature.

Among the many other directions of investigation, we mention the work of Hocevar [1980], who proposed measures of intelligence as predictive of creative accomplishments. In line with this, the psychology of science of [Feist, 1999, 2006, p.175] investigated which traits make scientific achievement more likely, which is interpreted as a creative outcome. He summarized the distinguishing traits of creative scientists by stating that "they are generally more open and flexible, driven and ambitious, and although they tend to be relatively asocial, when they do interact with others, they tend to be somewhat prone to arrogance, selfconfidence, and hostility." The fact that creative individuals are usually characterized by out-of-the-schemes psychological profiles is confirmed by Eysenck [1993], who made a connection between psychoticism and creativity.

A thorough analysis of the links between personality traits and the creative process is provided by Puccio and Grivas [2009]. They relied on the intuition that the creative process is made of four consecutive connected stages, namely problem clarification, idea generation, solution development and implementation planning. They explored the relationship between individuals' relative preferences for these stages and their personality traits. They found that different traits correlate with different preferences over the four stages of the creative process. For example, problem clarification turns out to be associated with tendencies to be cautious, careful, analytical, accurate and tactful, while idea generation is strongly preferred by subjects with personal traits as willingness to challenge prevailing thought, need for change and attraction to variety. These results question the robustness of previously detected correlations between specific personality traits and creativity. Indeed, the multidimensional nature of creativity makes it difficult to assess the impact of specific traits, since this might depend on which of the aforementioned stages has more objective weight in the creative process.

\subsection{Measurement of creativity}

One of the most controversial issues in creativity research is the measurement of the construct. This represents our second pillar of research on creativity in psychology. Before Amabile and Simonton's "revolution", existing approaches to measurement of creativity moved from personality tests and biographical inventories, to some more sophisticated behavioral measures. As a leading example, the works by Guilford [1950] suggested multidimensional criteria for interpreting variations in creativity, including sensitivity to problems, fluency, flexibility, originality, complexity, synthesizing, analyzing, reorganizing or redefining, and evaluat- 
ing. Still by Guilford [1975], in his structure-of-intellect model, creativity was defined as a form of problem solving, in which we can distinguish between two types of cognitive operations: divergent thinking and convergent thinking. Guilford highlights that the divergent production process is more relevant to successful creative thinking. In fact, divergent thinking is as a broad search used in open problems to generate logical answers or alternatives. Convergent thinking is instead a focused search that leads to the generation of a specific logical imperative for a problem, in which a particular answer is required.

The divergent-convergent thinking distinction was introduced by Guilford as a primary selection for assessing creative production, as different criteria apply to each category. It is now at the basis of the design of the tasks for measuring creativity not only in psychology, but also in management and economics research. On the same line, many other tests for assessing creative thinking have been developed, including the Torrance tests of creative thinking, introduced by Torrance [1966]. This test, which was strongly built upon Guilford's divergent criteria, represents the most used and referenced creativity test, in many contexts that go well beyond mere academic research. It includes game-like written and drawn tasks, and provides a sub-score for each creative characteristic assessed; sub-scores are then combined into a single creativity evaluation. The last updated version of the Torrance tests consists of six different criteria: fluency, originality, elaboration, abstractness of titles, resistance to premature closure, and creative strength.

In contrast to this predominant behavioral approach, the works of Amabile and Simonton went into two alternative directions. Simonton favored a quantitative approach [see Simonton, 1999]. His approach is based on the use of archival data on well-known creative individuals in history (such as classical components or scientists). He constructed variables from these existing records, such as word-counts of biographical dictionary entries for individuals in various fields, to estimate expert subjective assessments of the work of various creators (living and dead). Amabile, instead, rooted in a consensual operational definition of creativity [see Amabile, 1982a]. According to her consensual assessment technique, a construct is creative to the extent that some expert raters independently agree upon this judgment. This technique applies to sufficiently open-ended tasks that do not require special skills for which there is a a wide variation in the target population. Judges should be external observers with experience in the target domain, and should use their own subjective judgments of creativity to independently rate the outputs relative to one another, with an acceptable inter-judge reliability fixed at 0.70 or higher. The argument of Amabile is that creativity is subjective, so should be the assessment. The consensual assessment technique turned out to be really convenient as it overcame the difficulty of defining ultimate objective criteria for creativity. It represents now the most widely applicable method for assessing creativity in experimental research carried out by 
psychologists and economists.

\subsection{Intrinsic and extrinsic motivation in creative behavior}

The example of the Millennium Prize Problems discussed at the end of Introduction shows the crucial role of motivation in creativity generation. The starting point of the psychological research on creativity and motivation has been the analysis of the experience of subjects widely recognized for their creative work. In their introspection, well-known creative individuals have shed some light on how motivation arises, in particular on how personal will and curiosity or external factors may influence it. Two well representative examples proposed by Amabile [1982a, 1985] clarify this point. First, the poet Sylvia Path ascribed the cause of her own writer's block to her excessive concern with external recognition of her work. Second, an anecdote about Albert Einstein, who attributed a lean moment in his scientific curiosity to the imposition of school exams. Both cases underline a very important issue in research on creativity, that represents our third pillar, which sees extrinsic motivation as opposed to intrinsic one. Intrinsic motivation arises from the individual's perceived value of engaging in the task itself and the desire of being creative (e.g., finding it interesting, enjoyable, satisfying, or positively challenging). Extrinsic motivation comes from outside sources and it might be the response to an external demand (e.g., the promise of rewards or praise, or the threat of failing to meet a deadline or receiving a negative evaluation). The two aforementioned examples suggest the idea at the basis of one of the cornerstones of the research on creativity, which was articulated by [Amabile, 1985, p.2,5] as the motivation hypothesis of creativity: "the intrinsically motivated state is conducive to creativity, whereas the extrinsically motivated state is detrimental [...] In short, extrinsic motivators and extrinsic constraints in the social environment could alter motivational state from intrinsic to extrinsic and, thus, undermine creative behavior."

A relevant part of the psychological literature on creativity has been devoted on reinforcing the evidence that extrinsic motivation, in the form of expected evaluation or monetary rewards, may crowd-out intrinsic one [Condry, 1977, Tegano et al., 1991, see, e.g.,]. Some other works, such as those of Eisenberger et al. [1998, 1999] and Eisenberger and Rhoades [2001], went instead against the tide, coming to the conclusion that stating that reward always decreases creativity could have been premature. In their experiments with preadolescent schoolchildren, they found many scenarios in which the effect is totally opposite. For example, they showed a positive effect of incentives when people are trained to think divergently, when instructions explicitly convey the necessity of creative performance, or when people are performing tasks 
after having already been rewarded in a previous task.

Another external factor which has been proven to undermine intrinsic motivation in creativity is the so called fixation effect, i.e., the phenomenon according to which some knowledge about existing or obvious solutions may constrain the generation of original ideas while facing a creative task. In Agogué et al. [2014a], the authors relied on the most recent development in design theory to model the fixation phenomenon, namely the concept-knowledge design theory [Agogué et al., 2014b]. They test their theory through an experiment, showing that strong fixation effects exist in creativity and that participants usually give solutions based on common knowledge that is spontaneously activated.

\subsection{Individual vs team creativity}

Would anyone know Mark Zuckerberg today, if he got a single room at Harvard, 16 years ago? ${ }^{2}$ Or, would "The Starry Night" even exist, if Vincent van Gogh was an only child, without his younger brother Theo? ${ }^{3}$ These examples are meant to introduce the fourth and last "pillar" of our review of the research on creativity in psychology: team creativity and the importance of the so called "brainstorming" in the creativity process, as opposed to the "solitary genius" myth.

We have already mentioned in the Introduction how Amabile's reflection about the role of external factors in creativity resulted in what she called the componential theory of creativity. In this theory, next to the intra-individual components, there is an external component playing a crucial role: the social environment. Psychology research on creativity has spent many energies in testing and enlarging this theory, in particular with the aim of identifying more clearly in which form the social environment could play a role (e.g., previous experience, or additional training).

One of the most interesting developments in this direction concerns the discovery of team-level creativityrelevant processes, that, with a misuse of definition and given the diverse nomenclature in the literature, we will simply refer to as team creativity. The basic assumption when employing teams for problem solving is that group working usually results in a better outcome than that achieved by the same individuals alone, as ideas are generated by taking advantage of others' perspectives and skills. On this specific topic, the psychological literature borrowed some ideas from some old concepts which were already widespread in management research. In fact, it is up to Osborn [1953], an advertising executive, the definition of the term

\footnotetext{
${ }^{2}$ Mark Zuckerberg launched the Facebook social networking service from his dormitory, together with his college roommates Eduardo Saverin, Andrew McCollum, Dustin Moskovitz, and Chris Hughes.

${ }^{3}$ Theo van Gogh, who was an art expert, supported emotionally his brother, regularly encouraging Vincent's sketches and ideas for paintings, eventually providing him with advices.
} 
"brainstorming," as technique of listing all ideas put forth by a group in response to a given problem or question. Even if it is only in the 60s' that this term was popularized, the concept was in the mind of Osborn since a while and in the air since much longer, as the brainstorming technique boasts prestigious roots already in the ancient roman quaestiones. Since then, it became a well-stated practice for organizations to put tasks that require a problem solving approach in the hands of a group, rather than of a single individual.

The follow-up psychology research on team creativity remained pretty often on the borderline with the managements studies, mainly focusing on the question of how to compose the best performing group. In this regard, Taggar [2001, 2002] and Taggar and Neubert [2004] showed that group creative performance increases exponentially with the number of highly creative group members only when average creative ability of group members is relatively low. In other words, heterogeneity in individual creative abilities is a necessary condition for group working to boost creativity. This let us think that cooperation among group members with different creative abilities is another necessary condition. The latter is confirmed by Bechtoldt et al. [2012], who implemented a brainstorming task where groups with collectivistic value orientation generated more ideas than groups with individualistic value orientation. In fact, the former prioritize collective interest and seek cooperation, whereas the latter prioritize their self interest to group interest and try to outperform others in the group.

\subsection{Management studies on creativity and the road to innovation}

As we have mentioned in the Introduction, the influence from management to the research on creativity in psychology was already deeply present since the earlier studies. However, it is only in the 90s' that the discipline of management has started inquiring creative behavior on a regular basis, as a path to induce organizational innovation, and, consequently, firms' development and success. If creativity can be defined as the production of novel and useful ideas in any domain, innovation instead represents the successful implementation of these creative ideas within an organization [Charness and Grieco, 2019].

In brief, for creativity to become innovation, innovators should convince the market of the validity, plausibility, originality and novelty of their ideas. The two concepts seem necessarily linked (if not overlapping and often confused in a misuse of definitions). However, the measured correlations between innovation and creative performances from an experimental point of view is often mitigated, as shown by Bäker et al. [2011], who conclude that, certainly, other factors apart from creativity are crucial for innovation behavior [see, e.g., Cohendet et al., 2010, 2014]. However, regardless of the separate research traditions of psychology-based 
creativity and of management-based innovation, researchers in management realized how these two disciplines need to be combined for a better understanding of innovation behavior. This combination has been easily allowed by the use of the experimental methodology in and outside the laboratory. Slavich and Svejenova [2016] tried to organize this research link, suggesting that the principal direction lines that stimulate management research on creative behaviour are represented by three dualities. Here we extend their analysis by including four additional trade-offs that come from the psychology literature on which the most recent managerial studies of creativity rely. In Table 1, for each of the dualities in the extended list, we indicate representative experimental studies in management which investigate the specific issue.

Table 1: Dualities in creative behavior analyzed in management studies on creativity

\begin{tabular}{|l|c|}
\hline Dualities & Experimental studies \\
\hline \hline Individual vs collective creativity & Chen et al. [2012], Taggar [2002] \\
\hline Permanent vs temporary creativity & Kachelmeier et al. [2015], De Fillippi et al. [2007] \\
\hline Creative outcome vs process & Williams [2004] \\
\hline Convergent vs divergent thinking & Vincent and Kouchaki [2016] \\
\hline Intrinsic vs extrinsic motivation & Dewett [2007], Gerhart and Fang [2015], Gross [2018], Baer et al. [2003], Kachelmeier et al. [2015] \\
\hline Dependence vs independence from personality traits & Bouncken et al. [2015], Chua et al. [2015] \\
\hline Dependence vs independence from cultural factors & \\
\hline
\end{tabular}

More in general, each of the trade-offs in Table 1 also shape the directions of analysis of creativity in experimental economics, which we will present in details in the following sections.

\section{Economic Experiments on Creativity}

\subsection{The role of incentives}

As we have already extensively discussed in the Introduction, it is widely recognized that creativity represents a main driver of the world's economy and that, as such, it is valuable to stimulate it to the extent possible [see, e.g., Romer, 1992, Aghion and Howitt, 2008, Phelps, 2013]. Then, what factors are more likely to nurture it? With the aim of providing a response to this question, most of the literature in economics on creativity has focused on the role of incentives [see, e.g., Toubia, 2006, Azoulay et al., 2011, and the references therein]. We have also anticipated in the Introduction that, due to the need of collecting empirical evidence and the scarcity of field data, the natural way to perform objective measurements of creativity and clean tests of the link between incentives and creativity has turned to experimental economics, i.e., the implementation of controlled laboratory experiments. This is the reason why in the part of this survey dedicated to economics research on creativity (Sections 3 and 4), we narrow our field of interest to contributions in the domain of experimental economics. 
The intuitive logic for providing incentives is to increase the efforts of the individuals, giving them a motivation to focus on succeeding in a given task. Incentives in economics are usually in the form of a monetary reward. The role of monetary incentives and their extrinsic motivation effects have been largely studied in theoretical economics [see, e.g., Kreps, 1997]. However, in contrast with the classic microeconomics labor supply theory, according to which people provide more effort under some kinds of performance-based compensation irrespective of the task, several field examples show that incentives may not be necessary (e.g., artists and researchers, who do not appear to need financial incentives to be productive) or even be counterproductive (e.g., basketball players, who perform better during training than during an official game; see Dandy et al. [2001]). Building on real-world examples, the theoretical literature has started to model nonmonetary incentives to effort provision, such as social norms Kreps [1997], or boosting of self-confidence by a second party [Bénabou and Tirole, 2003], to cite two of the most well-known frameworks.

The study of incentives in laboratory experiments has often focused on the trade-off between monetary and non-monetary incentives [for a survey, see Erkal et al., 2018], providing mixed evidence on the relationship between monetary incentives and effort provision. If, on one side, a large experimental literature finds a positive impact of incentives on effort, it is often neglected that working on some tasks may be in itself rewarding and people might be intrinsically motivated. As already previously observed by the large literature on psychology (see Section 2.3), this may be specifically true for creativity tasks, in which we may expect extrinsic incentives to crowd out intrinsic motivation and therefore not lead to the intuitive result of creativity boosted by monetary reward.

Differently from the psychological literature, in which extrinsic incentives are usually proposed in the form of an evaluation which does not imply a monetary reward, the experimental economics approach crucially relies on the saliency of monetary incentives in enhancing the revelation of the subjects' true preferences. The experimental economics studies on creativity also adopted the standard rule of salient monetary incentives. In Table 2 we present a summary of the monetary incentives implemented in the experimental economics studies of our review, with details about the size of the payments, and their average and maximum value across experimental participants. With the aim of increasing comparability among the different works in terms of relative strength of monetary incentives, we also provide information about: minimum wage per hour in the country and at the time the experiment was performed; the currency used for the payments and the purchasing power parity according to the currency-country combination; the average duration of the experiment; the number of experimental participants. It is not surprising that, given the newborn interest of the experimental literature on this creativity topic, we have only found 13 working papers or published articles 
which satisfy the basic principles and minimum requirements of experimental methods in economics: controlled environment, salient monetary incentives, participants' anonimity, unbiased sample of participants, etc.

Table 2: Monetary incentives

\begin{tabular}{|c|c|c|c|c|c|c|c|c|c|}
\hline & ह & 语 & $\nabla^{\infty}$ & से & इ & $0^{8}$ & 2 & 结 & क्षे \\
\hline Ariely et al. [2009] - Exp. 1 & $\bar{x}$ & $\bar{x}$ & - & $\$ \$ 0.05, \$ 5.22$ & $\$ 0.61$ & Rs & $\begin{array}{ll}\text { Rs9.68 } \\
\end{array}$ & $\overline{-1}$ & $\overline{87}$ \\
\hline Ariely et al. [2009] - Exp. 3 & $\mathrm{x}$ & & - & - & $\$ 7.25$ & $\$$ & $\$ 1.00$ & $30 \mathrm{~min}$ & 39 \\
\hline Attanasi et al. [2019a] & $\mathrm{x}$ & & $\$ 17.60$ & $\$ 21.60$ & $\$ 10.86$ & $€$ & $€ 0.75$ & $75 \mathrm{~min}$ & 240 \\
\hline Attanasi et al. [2019b] & $\mathrm{x}$ & & $\$ 17.60$ & $\$ 21.60$ & F: $\$ 10.86, \mathrm{~V}: \$ 4.25$ & $€$, VND & F: $€ 0.75$, V: VND8.07 & $75 \mathrm{~min}$ & 240 \\
\hline Boudreau and Lakhani [2011] & $\mathrm{x}$ & & $\$ 50.00$ & $\$ 500.00$ & $\$ 7.25$ & $\$$ & $\$ 1.00$ & 10 days & 40 \\
\hline Bradler [2015] & $\mathrm{x}$ & & $\$ 23.08$ & $\$ 28.03$ & $\$ 9.24$ & $€$ & $€ 0.78$ & $90 \mathrm{~min}$ & 130 \\
\hline Bradler et al. [2016] & $\mathrm{x}$ & & $\$ 16.25$ & $\$ 29.13$ & $\$ 9.24$ & $€$ & $€ 0.75$ & $75 \mathrm{~min}$ & 1123 \\
\hline Charness and Grieco [2013] & $\mathrm{x}$ & & $\$ 15.00$ & $\$ 40.45$ & $\$ 7.25$ & $\$$ & $\$ 1.00$ & $60 \mathrm{~min}$ & 236 \\
\hline Charness and Grieco [2014] - Exp. 2 & $\mathrm{x}$ & & $\$ 15.00$ & - & $\$ 7.25$ & $\$$ & $\$ 1.00$ & $25 \mathrm{~min}$ & 174 \\
\hline Charness and Grieco [2019] & $\mathrm{x}$ & & $\$ 15.00$ & $\$ 40.45$ & $\$ 7.25$ & $\$$ & $\$ 1.00$ & $60 \mathrm{~min}$ & 328 \\
\hline Dutcher and Rodet [2018] & $\mathrm{x}$ & & - & - & - & - & - & $28 \mathrm{~min}$ & 66 \\
\hline Eckartz et al. [2012] & $\mathrm{x}$ & & $\$ 11.17$ & $\$ 32.36$ & $\$ 9.24$ & $€$ & $€ 0.78$ & $60 \mathrm{~min}$ & 216 \\
\hline Erat and Gneezy [2016] & $\mathrm{x}$ & & - & $\$ 26.95$ & $\$ 7.25$ & $\$$ & $\$ 1.00$ & $10 \mathrm{~min}$ to 1 hour & 257 \\
\hline Laske and Schröder [2017] & $\mathrm{x}$ & & $\$ 15.64$ & - & $\$ 9.61$ & $€$ & $€ 0.74$ & $40 \mathrm{~min}$ & 126 \\
\hline Mohnen and Ostermaier [2013] & $\mathrm{x}$ & & $\$ 15.85$ & - & $\$ 9.24$ & $€$ & $€ 0.77$ & $60 \mathrm{~min}$ & 136 \\
\hline Saad et al. [2015] & $\mathrm{x}$ & & $\$ 10.00$ & - & C: $\$ 7.38, \mathrm{~T}: \$ 4.66$ & CAD, TWD & CAD1.25, TWD14.84 & $45 \mathrm{~min}$ & 256 \\
\hline
\end{tabular}

Currency abbreviations: Rs (Indian Rupees), VND (Vietnamese Dongs), CAD (Canadian dollars), and TWD (Taiwanese dollars). Country abbreviations: F (France), V (Vietnam), C (Canada), and $\mathrm{T}$ (Taiwan). The Purchasing Power Parity (PPP) is the rate of currency conversion that tries to equalize the purchasing power of different currencies, by eliminating the differences in price levels between countries; it is expressed in national currency units/US dollar, 2000-2019 data from OCDE, link: https://data.oecd.org/conversion/purchasing-power-parities-ppp.htm. Observe that the higher payment of $\$ 5.22$ in the first experiment of Ariely et al. [2009] corresponds to $400 \mathrm{Rs}$, which is almost equivalent to rural India average monthly per capita consumer expenditure (which was of Rs 495 at the time of the experiment). For this reason, this payment is classified as "high".

\subsection{Creativity tasks}

Finding a task for measuring creativity may turn out to be quite complicated. Requirements may vary from the need of easily assessing the quality of a solution, to the possibility of repeating the task for the same subject more than once. Depending on the final goal of their analysis, researchers in experimental economics have been very creative themselves in designing different settings to enhance creativity. We may classify these tasks in two main groups, based on whether they are intended to mimic a closed or an open creativity problem. A closed creativity problem is stated with a specific and delineated goal (e.g., developing a new drug or vaccine for a specific disease). In the experimental implementation, it translates into a task that is presented to the participants, when the method for solving the problem is known, and when the correct response is unique (for this reason, it may be also referred to as convergent thinking task according to the terminology of Guilford [1975], presented in Section 2.2). Closed tasks are usually easy to assess, but most of the times they can be used for each participant only once, because once the solution is revealed, the problem loses interest. Closed creativity overlaps with the definitions of experimental creativity in Galenson 
[2004]. On the contrary, an open creativity task allows for thinking outside the box, without any ex ante direction (this is the case, for example, of the production of a piece of art work). In the corresponding experimental task, the subject is required to find, invent, or discover the problem, and the correct answers may be various and different (this may be referred to as divergent thinking task according to the terminology of Guilford [1975]). Open creativity overlaps with the definitions of conceptual creativity in Galenson [2004]. Open tasks have the advantage of remaining interesting for a subject even after some repetitions. However, it is often difficult to judge the creativity of the proposed solution, apart from some examples in which an objective mechanical evaluation is possible.

Once stated the distinction between closed and open creativity, we should remember that there may exist many nuances in between, depending on the autonomy we let to individuals while performing a task. For example, Charness and Grieco [2013] distinguish between in box (replaced with the term "closed" in Charness and Grieco [2019]) and blue sky ("open”) creativity. However, the latter perfectly represents the open condition, under which pure and unfettered thinking outside the box is allowed, without any suggestion of direction. In box creativity, on the other side, provides a specific goal with constraints, but correct answers may be variegate, as the methods for finding them. As such, in our classification the in box creativity $\grave{a} l a$ Charness-Grieco is placed at a middle point between the two classical definitions of closed and open tasks, and we refer to it as an "open with constraints" task. In Table 3 we present again our list of experimental papers in economics, specifying for each of them the type of implemented creativity task, and our classification in terms of open, open with constraints, and closed creativity. We may observe that the implementation of completely open tasks is pretty rare, while most of the experiments are built on some sort of constraints.

\subsection{Payment schemes, group interaction, and creativity assessment}

Given a certain amount of monetary incentives and once a task has been chosen, the implementation of an experiment on creativity with monetary incentives should take into account at least other three dimensions: the rule behind the incentives provision (the payment scheme), the possible interaction with other individuals, and the way (individual or group) creativity is assessed. These three design features are strictly connected, since, as long as monetary incentives are in the picture, the experimenter has to set an individual payment scheme, whether and how this rule depends on the behavior of other individuals in the experiment, and how the value of the (creative) outcome is assessed in order to link it to the payment scheme. The analysis about the investigation on these three dimensions in the papers of our review is presented in Table 4. 
Table 3: Creativity tasks

\begin{tabular}{|c|c|c|c|c|}
\hline \multirow{2}{*}{ Paper } & \multicolumn{4}{|c|}{ Creativity task } \\
\hline & type & open & open with constraints & closed \\
\hline Ariely et al. [2009] - Exp. 1 & Packing & & & $\mathrm{x}$ \\
\hline Ariely et al. [2009] - Exp. 3 & Anagrams & & & $\mathrm{x}$ \\
\hline Attanasi et al. [2019a] & Verbal, maths, draw & & $\mathrm{x}$ & \\
\hline Attanasi et al. [2019b] & Verbal, maths, draw & & $\mathrm{x}$ & \\
\hline Boudreau and Lakhani [2011] & Alghoritm & & & $\mathrm{x}$ \\
\hline Bradler [2015] & Torrance test of creative thinking & & $\mathrm{x}$ & \\
\hline Bradler et al. [2016] & Torrance test of creative thinking & & $\mathrm{x}$ & \\
\hline Charness and Grieco [2013] & Verbal, maths, imagine, describe & $\mathrm{x}$ & $\mathrm{x}$ & \\
\hline Charness and Grieco [2014] Exp.2 & Verbal, math, draw & $\mathrm{x}$ & $\mathrm{x}$ & \\
\hline Charness and Grieco [2019] & Verbal, maths, imagine, describe & $\mathrm{x}$ & $\mathrm{x}$ & \\
\hline Dutcher and Rodet [2018] & Torrance test of creative thinking & & $\mathrm{x}$ & \\
\hline Eckartz et al. [2012] & Scramble & & & $\mathrm{x}$ \\
\hline Erat and Gneezy [2016] & Rebus & $\mathrm{x}$ & & \\
\hline Laske and Schröder [2017] & Illustrate words & & $\mathrm{x}$ & \\
\hline Mohnen and Ostermaier [2013] & Scramble & & & $\mathrm{x}$ \\
\hline Saad et al. [2015] & Brainstorming & $\mathrm{x}$ & & \\
\hline
\end{tabular}

As for the payment scheme, we identify three main rules for inducing extrinsic motivation in subjects performing a creativity task: flat-payment, performance-based, and tournament. With a flat-payment scheme, also referred to as fix payment, fixed-wage or flat fee, we denote the same payment for each subject, independently of his/her performance. Conversely, a performance-based payment rewards the subject depending on his/her achievement in terms of creativity. In this category we also include the standard piece-rate payment, where the monetary reward positively depends on the number of fulfilled tasks. Under the tournament category, instead, we list all experiments in which the payment does not only depend on the individual performance, but also on the relative achievement of other individuals. Note that this dimension is crucial in the creativity elicitation, as in most of the experimental studies included in our review, the research question is strictly focused on the effects of different kinds of incentives (e.g., flat-payment vs performance-based). Some of the studies also introduce a self-selection stage, in which the subjects may choose their preferred incentive scheme.

Second, as for subjects' interaction in the creativity task, recall that the trade-off between individual and group creativity is a pillar in the psychological literature (see Section 2.4) and it became the crucial point for investigation on creativity in management (see Section 2.5). In this regard, the experimental economics literature studies the effects of a competitive and/or a cooperative environment withing a group. Experimental works investigating the competitive and the cooperative settings are discussed in Sections 4.3 and 4.4, respectively. Differently from the literature in psychology and management, however, experimental 
Table 4: Implementation of monetary incentives: design features

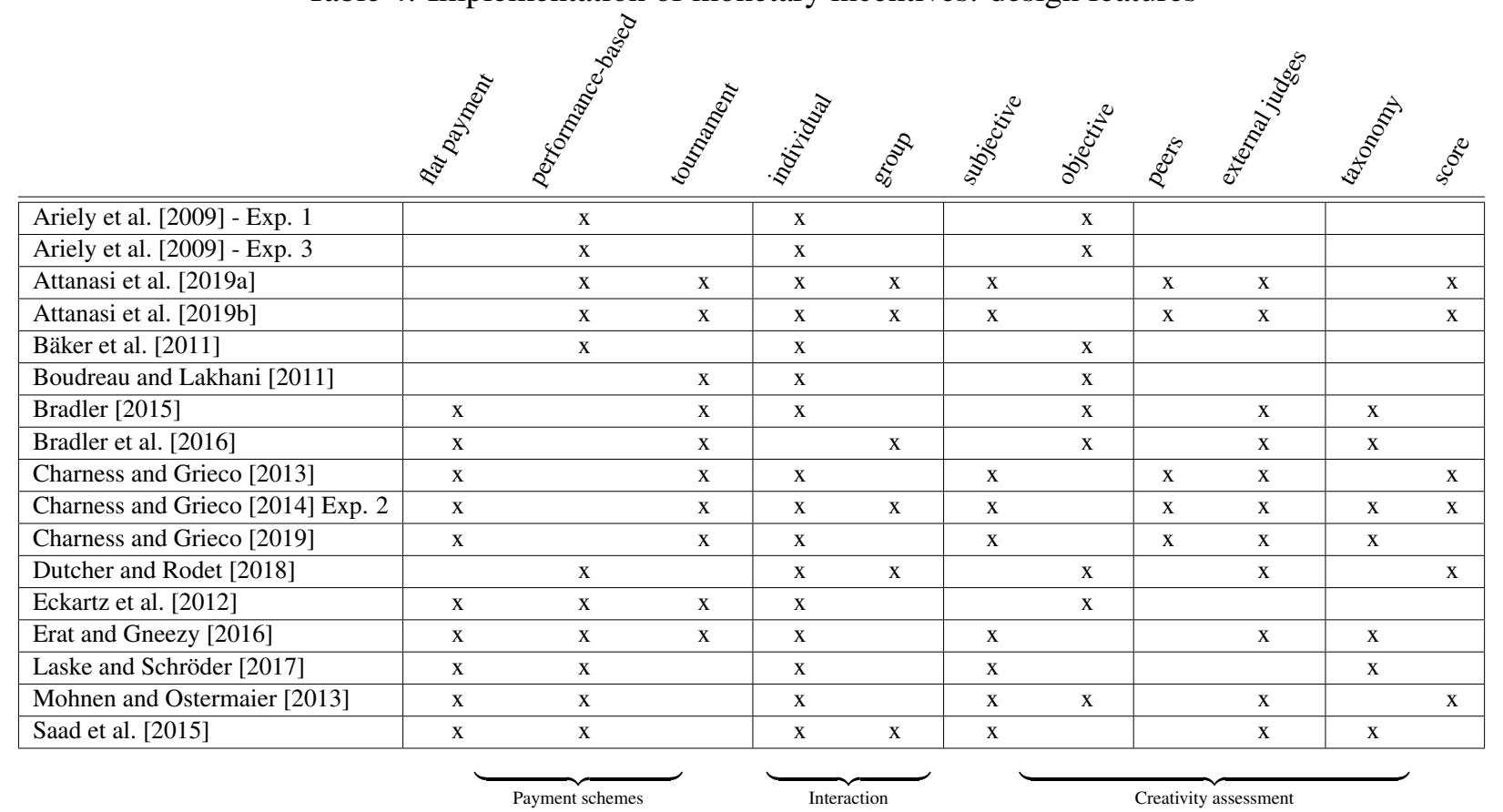

studies in economics mainly focus on the individual level of creativity resulting from group interaction, rather than on the group performance.

Finally, as for the creativity assessment, recall that when we discussed it as one of the pillars in the psychological literature in Section 2.2, we referred to Guilford [1975] convergent vs divergent thinking trade-off. Guilford substantially states that different criteria apply to different tasks. This logic also applies to the economic experimental literature, where to a chosen task corresponds a specific creativity assessment. In particular, closed tasks are usually judged according to objective rules by the experimenters or by a computer, while open or open with constraints ones are usually characterized by a subjective assessment of the performance. In this last case, the pool of judges may be represented by peers, i.e., a group of subjects performing the same task, or external judges, which might be experts of creativity, academic colleagues of the experimenters, a larger group of students not involved in the experiment, or a broader online pool. When implementing a subjective evaluation, moreover, we can make a further distinction: the one based on a taxonomy, intended to isolate different aspects of creativity, and a score (usually from 1 to 10). The latter, in particular, is fully in line with Amabile's consensual assessment technique, according to which raters should be capable of independently agreeing upon a creativity judgment, without defining ultimate objective criteria for creativity.

The comparison between objective and subjective evaluation of creativity is the aim of Mohnen and 
Ostermaier [2013]. Relying on a Scramble-like task, they show that the two measurements are strongly correlated. However, in favor of objective evaluation, subjects tend to put more effort in the task than under a subjective evaluation. As a drawback, they discuss an interesting inconsistency: the rules of the score being previously subjectively decided by some experts, the very definition of "objective" is contradicted.

\section{An overview}

The aim of our study is to provide an overview of the main issues that works in experimental economics have tried to address on the topic of creativity. We now discuss more in depth the papers that we have found in this literature, which are listed in Tables 2-5. Given the complexity of organizing this discussion in a structured way, as each work is different in terms of e.g. tasks, payment schemes or subject pools, we decided to present this review by pointing out the primarily scope of each paper, as stated in the research question by its authors. We will try to make the link in between sections, whenever this is useful for the understanding of the reader.

As anticipated in the Introduction, we identified six main directions of analysis, namely, the impact on creativity of: (1) low vs high monetary incentives, (2) the interplay of monetary incentives and tasks, (3) within-group competition, (4) within-group cooperation, (5) cultural factors, and (6) non-monetary social incentives. For each of these directions, we highlight the works which are, in our opinion, particularly representative of the approaches and the results on the topic.

At the end of this section, we dwell into an additional direction of analysis: the role of the personality traits. This aspect represents the first pillar in our review of the psychological research on creativity of Section 2.2, and it is also a crucial aspect for the experimental economics investigation. However, it is always presented as an additional cue of analysis (in almost all the works), and it never represents the main research question. For this reason, we discuss it separately at the end of the section.

\subsection{Low vs high monetary incentives}

Ariely et al. [2009] address one of the fundamental questions of our survey: does increased monetary compensation always result in increased creativity? In the first experiment presented in this paper, they implemented six games in a rural town in India, including a packing quarters game to test what they call "primarily creativity," and that we can classify in the category of closed tasks. In this game, participants were asked to fit nine metal pieces of quarter circles into a black wooden frame within a given time. The 
difficulty of the task lies on the fact it is easy to fit eight pieces, in many different ways, but, to fit nine, the pieces have to be packed in a particular way. As reward for the performed task they received different monetary stakes: low, mid or high. The crucial idea of this research has been to conduct the experiments in India. In fact, this allowed the authors to put on the plate significant monetary incentives on a relative modest budget, such that the maximum possible payment in the high-stake treatment was relatively close to average monthly per capita consumer expenditure in rural Indian areas. Such stakes are effectively much larger than those that are typically offered in experimental settings. The comparison of the low and mid incentives levels revealed little difference in performance. Surprisingly, however, comparisons between the low-stake and the high-stake treatment revealed a number of statistically significant differences in favor of more creativity generated in the former, underlying the counterintuitive result that high monetary incentives can have perverse effect on performance. In this regard, the authors introduce the concept of chocking under pressure for creativity, i.e., raising contingent incentives beyond a certain threshold may be a losing proposition. This result may be read, according to the usual terminology, as a crowding-out effect of too high monetary incentives on creativity when performing a closed task. Surprisingly, this is, to the best of our knowledge, the only paper to attack the crowding-out problem through an exogenous variation of the stakes' size, which is however a standard method in experimental economics. Therefore, no further comparison to confirm or contradict Ariely et al. [2009] statement is available.

\subsection{Interplay of monetary incentives and creativity tasks}

In Section 3.2 we highligthed that there exist many different tasks which are designed to measure different kinds of creativity. Then, one crucial issue is to study how the effect of specific incentives varies, depending on the chosen task.

Charness and Grieco [2013] shed some light on this issue, by focusing on open tasks with vs without constraints. They analyze whether incentives act differently in stimulating creativity when implemented on different tasks. In particular, they implemented what they call an in box (open with constraints) creativity task, where ex-ante goals and constraints are imposed on the answers, and a blue sky (open) creativity task, in which no restrictions apply.

In the in box creativity task, subjects were asked to choose one out of the two tasks: "Choose a combination of words to create an interesting story. The words supplied are: house, zero, curve, relevance, cow, tree, planet, ring, send;" or, "Starting from the number 27, obtain the number 6 by using at least two different 
numerical operations. Possible answers include: $(27: 3)-3=6$, or $[(27+3): 2-12] !=6$." Although they could write the story they liked or use the mathematical operations they preferred, the task compelled the subjects to use a given set of words or to start/end with a given number, respectively. In the blue sky creativity task, subjects were asked to answer one out of the two questions: "If you had the talent to invent things just by thinking of them, what would you create?," or "Imagine and describe a town, city, or society in the future." Unlike the in box task, no constraint was imposed.

As for the payment scheme, each subject's creativity in his/her chosen task was assessed on a tournament base in relation to that of other four subjects in the experimental session. In one treatment, indepedently of the tournament ranking, each subject received a simple flat payment. In the other one, each subject was paid on the basis of the assessments of his/her own creativity. In both treatments, the five competing subjects were told that other five subjects in the experiment would have unanimously ranked the creativity of their task (peer ranking à la Amabile). Moreover, after the end of the experiment, subjects' creativity was also evaluated - through a 1-10 score - by two external judges blind to treatment.

Relying on the latter evaluation, the authors obtained that the introduction of monetary incentives has a significant positive effect on the level of creativity when implementing the in box condition. However, in the blue sky condition, the average creativity score with monetary incentives is not significantly different from that in the flat payment regime. The take-off lesson of this paper is that monetary incentives are particularly effective on tasks with reasonable clarity about goals and constraints. Absent such clarity (i.e., in a purely open creativity task), monetary incentives are ineffective, although there is no evidence of a crowding-out effect. The extended analysis of this issue in Charness and Grieco [2019], based on the same tasks and under similar conditions, confirms this result.

This work is in line with the suggestion of some previous contributions by Collins and Amabile [1999] and Sanjiv and Krishnan [2012], according to which monetary incentives might promote creativity in closed tasks, while, on the contrary, directly incentivizing open creativity would be ineffective or even counterproductive.

In line with these results is also the work of Laske and Schröder [2017]. Differently from the aforementioned papers, in this study the authors perform a multidimensional analysis, studying on the same task which dimensions of creativity are affected by monetary incentives and which dimensions are, in contrast, merely driven by intrinsic motivation and individual talent. They provide objective measures for three dimensions of creativity: quantity (number of ideas), quality (degree to which an idea is suitable for its intended purpose), and originality (degree of innovation of an idea). They find that performance-based in- 
centives significantly affect the quantity and average quality of ideas, but not the average originality. In particular, they observe that increasing quantity results in a quality decrease, with the consequent raise of a trade-off for organizations choosing to introduce incentives for creative performance.

\subsection{Within-group competition}

In this section, we present some works whose research question is focused on the impact of different payment schemes, with a particular attention on the effect of within-group competition. We present examples in which a tournament is implemented within a group, such that a subject's payment depends on his/her relative performance with respect to the other group members. We may observe that this way of ranking the subjects in order to obtain an evaluation of their performance is pretty standard and it is implemented in many different experiments on creativity (e.g., Charness and Grieco [2013, 2019] or Attanasi et al. [2019a,b]). However, in this section, we selected some works whose research question was particularly focused on comparing such method to some other payment schemes.

We begin by discussing three works under the same research question, whose results highlight the confusion of the debate on the topic: the first work finds no effect, the second one a negative and the last one a positive effect of tournaments on creative outputs.

The first work is by Eckartz et al. [2012]. This study considers a flat payement, a performance-based, and a tournament scheme. In the first part of the experiment, subjects faced the three schemes as imposed by the experimentalists, while in the second part they had the possibility of choosing their own preferred scheme. The task was in the form of a Scrabble-like game, in which participants were presented with an alphabetically ordered letterset, consisting of 12 letters. Participants had to construct as many and as long words possible within 5 minutes. In the flat payment, they received a fixed amount of money, irrespective of their performance. In the performance-based payment, they got for every correct word 1 point for the first letter, 2 points for the second one, and so on, and then these points were proportionally corrected into money. In the tournament, each participant was matched with three other participants and the performances (evaluated as for the performance-based scheme) compared. The winner was awarded a higher payment, and the three remaining subjects got a lower payment, in the form of a show-up fee as compensation for their effort. As final result, the authors did not find any effect of any kind of incentive and concluded that performances are predominantly related to individual skills.

In Erat and Gneezy [2016], the focus is still on the comparison of the same three payment schemes, this 
time implemented according to a between-subject design. The proposed task is a different one, consisting in inventing a rebus. A rebus is a puzzle made with words and/or picture with a hidden and non-obvious solution. In the flat payment case, subjects' creativity was evaluated with a score from 1 to 10 by a panel of judges, but they did not receive any additional payment depending on this evaluation. In the performancebased scheme, under the same assessment method, subjects received a fixed amount of $\$ 2$ for each point of score, on average across judges' score. In the tournament, each subject was paired with another subject, and the two average scores compared. Only the winner was awarded with $\$ 4$ for each point of score, on average across judges' score. In case of a tie, both of them received $\$ 2$ for each average point of score. Note that this tournament is much different from the one in Eckartz et al. [2012] as, in case of victory or tie, the payment depends on the individual performance, and not simply on the relative ranking. Betweentreatment comparisons on creativity assessments show that the performance-based scheme does not affect assessed creativity, compared to the flat payment. However, tournament competition has a detrimental effect on creativity, as it reduces the assessed creativity of the participants.

Bradler et al. [2016] report the results from a large-scale experiment investigating the impact of a tournament scheme on creativity. Subjects were randomly assigned to groups of five participants, with one of them playing the role of a principal, and the other four the role of agents. The four agents worked for three periods for their principal on a creativity task. The creativity task consisted in implementing the "unusual uses task" (a part of the Torrance tests of creative thinking in Torrance [1966]). In this task, participants are asked to name as many, unique and unusual uses for each of the three ordinary items: a sheet of paper, a tin can and a cord. This is an open task, but which provides a clean objective measure of creative production (based on counting the valid answers by subject as well as the statistical frequency of a given answer across subjects). The output of the principal is increasing in the assessed creativity of the agents' output. In a first treatment, during the three periods, the agents received a flat payment. In a second treatment, in addition to the flat payment, and only in the second period, the principal could decide whether or not to provide an additional reward to all the four agents (at his/her own expenses and independently of each agent's creative output) in the form of a gift. In a third treatment, instead, the principal could provide an additional monetary prize (at his/her own expenses) twice as big as in the gift treatment, but only to the $50 \%$ best performing agents. The authors found that tournaments have a large and statistically significant positive effect on the creative performances. By comparison, gifts were ineffective, as agents did not reciprocate the common gift in the creativity task.

In order to read the results of these three works, it is important to underline the variety of the tasks they 
proposed: closed, in the first case, and open, in the second and in the third examples. However, surprisingly, Erat and Gneezy [2016] report evidence of crowding out of intrinsic motivation, due to competition, while Bradler et al. [2016] conclude the opposite, showing how creativity can be boosted by a competitive environment.

We conclude this section by discussing two works which are still focused on the effects of competition, but whose research question is slightly different from the one of the previous three papers. In the first one, Bradler [2015] proposes an experiment in which the subjects were asked to make a choice about whether to perform a task under a flat-payment, or a tournament scheme. This research question is similar to the one already investigated by Eckartz et al. [2012] in the second part of their experiment. In their paper, however, subjects had also the additional choice of opting for a performance-based scheme and the choice could be made after having already experienced the three different schemes. Instead, in Bradler [2015] the choice had to be made prior to having practiced on the two different options. According to her results, and differently from the findings of Eckartz et al. [2012], tournament schemes seem to foster performance in creativity tasks, but, surprisingly, they do not necessarily attract the most creative subjects.

In the second one, Boudreau and Lakhani [2011] report the findings of a 10-day field experiment, involving more than 500 elite programmers engaged in trying to create a software solution to a real computational engineering problem from NASA. They study the effects on creativity when implementing a tournament within a group of subjects with declared strong preferences on working in a competitive environment (sorted) vs implementing the same tournament in a group of (randomly) unsorted ones. Their main finding is a significant effect in favor of average creativity of subjects in the sorted group, showing that their performances are nearly double than those of subjects in the unsorted group.

\subsection{Within-group cooperation}

As for within-group competition, also within-group cooperation is a very common organizational mode in creativity in the psychological and management literature. The experimental economics literature focuses one more time on the problem by paying a particular attention to the role of the monetary incentives in a cooperative environment. However, the production on this research question is still limited.

Charness and Grieco [2014] proposed a follow-up of the previously presented Charness and Grieco [2013], studying group creativity in contexts where corporate culture may tend to promote cooperation among group members. We may observe that the 2014 version of the paper also included the individual 
analysis that was then separately published in Charness and Grieco [2019]. Then, here we simply focus on the cooperative analysis which represents the second part of this paper (referred to as Exp. 2 in the tables). The tasks of this paper were three, namely: verbal, maths and draw. The first two ones were borrowed from Charness and Grieco [2013], that we already presented in Section 4.2 as in box (open-with-constraints) tasks. The third one was stated as: "Draw a picture using any combination of shapes you like: the only constraint you have is that you must use all the following shapes" (and then a picture was showing 9 shapes: 3 triangles, 3 squares and 3 circles). The authors implemented four treatments in a $2 \times 2$ design: "individual corporate culture" vs "cooperative corporate culture" and "financial incentives" vs "no incentives". For all the treatments, the creative assignment was still individual, but each subject was allocated to a triplet, i.e., a set of 3 subjects, each one having a different assignment (verbal, maths, or draw). Subjects in the same triplet were sitting close to each other and had the possibility of talking and helping each other and even of switching tasks with each other. The two individual corporate culture treatments were the same as in Charness and Grieco [2013, 2019], either payed according to the individual performance, $\$ 15$ if first, $\$ 12$ if second, etc. (in case of financial incentives), or with a flat-payment of $\$ 9$ (in case of no-incentives). In the cooperative corporate culture and financial incentive treatment, people were payed on the basis of the group's assessed performance. Participants answering each task (verbal, math or draw) were ranked and then these ranks were summed for the three group members. Each person in the group with the best ranking won $\$ 15$, each person in the group with the second-best ranking received $\$ 12$, and so on. Finally, in the cooperative corporate culture with no incentives, subjects were payed a flat-payment of $\$ 9$, but a feedback about the individual and the group ranking was provided. As a results of their analysis, the authors report that financial incentives are effective only when the environment rules promote cooperation among co-members. On the contrary, they are ineffective when the subjects perform individually (because of a crowding-out effect of intrinsic motivation in case of incentives without cooperation).

In a very recent work, Attanasi et al. [2019a] built on the same experimental design of Charness and Grieco [2014], with the just mentioned open-with-constraints tasks (verbal, maths and draw), but designing some different treatments for analyzing the interplay between monetary incentives and group cooperation. The key point was to introduce a novel model of intrinsic vs extrinsic motivation to group collaboration in creativity and to test if there is a crowding-out effect of intrinsic motivation due to extrinsic incentives to group creativity. For each of the three open-with-constraints tasks, Attanasi et al. [2019a] implemented four treatments: Individual, Group-no, Group-cooperation, and Group-competition. The Individual treatment was the same as in Charness and Grieco [2013, 2019]. Differently from Charness and Grieco [2014], in 
the individual treatment subjects were isolated and not able to communicate among them. For all the group treatments, the creative assignment was still individual, but each subject was allocated to a triplet, as in Charness and Grieco [2014], but with the only difference that subjects were not allowed to switch tasks with each other. Then, in the Group-no treatment, individual earnings were as in the Individual treatment. In the Group-cooperative, the individual earning was the average of the triplet's earnings ${ }^{4}$. Finally, in the Group-competition, individual earnings were determined through within-group competition over the group earnings obtained in the three separate tournaments. As in Charness and Grieco [2013, 2019], individual creativity is assessed both by peers - during the experiment - and by external judges - after the end of the experiment.

The results of this work outline some interesting hints on extrinsic motivation to group collaboration. In particular, group collaboration in creative assignments with no monetary incentives for the group (Groupno treatment) significantly increases creativity compared to individual work (Individual treatment). The authors assign this effect to intrinsic motivation to be creative being boosted by group working - intended as organizational mode -, without the need of monetary incentives. ${ }^{5}$ Conversely, monetary incentives to group collaboration (Group-cooperation treatment) do not increase creativity compared to individual work. This result is interpreted as extrinsic motivation to group collaboration crowding out the intrinsic one. Finally, monetary incentives to group competition (Group-competition treatment) significantly increase creativity compared to individual work, showing that even if extrinsic motivation to cooperate is detrimental to intrinsic motivation, group extrinsic motivation to compete is not.

The work of Dutcher and Rodet [2018] investigates how diversity within a group affects the creative performance. The main idea of the paper is that groups may benefit from members with diverse sets of experiences, which come from social learning, formal learning, and/or belonging to different demographic groups. The task is the common "unusual uses task" (that we have already presented for Bradler et al. [2016] in Section 4.3), but with the following set of objects: a brick, a cardboard box, a metal pipe, and a t-shirt. The experiment was organized over four periods. In the first period, subjects worked alone and were payed based on their own output: $\$ 0.15$ for every valid submission and another $\$ 0.15$ for each unique (and valid) submission. In periods 2-4, subjects sat side-by-side with one partner and submitted creative uses into a single computer terminal. Each group member was paid $\$ 0.15$ for every valid submission and

\footnotetext{
${ }^{4}$ Note as this is much different from the cooperative design in Charness and Grieco [2014], in which subjects were payed according to the group ranking and not according to the average of their individual earnings.

${ }^{5}$ This result is in line with some previous works on creativity and cooperation. For example, Baloche [1994] implemented experiments with students of music in an elementary class, showing how cooperative learning can positively affect creativity.
} 
$\$ 0.15$ for every unique submission that the pair came up with. Complementary to the experimental task, the subjects had to complete a questionnaire that provided measurements of group diversity. The first part of the questionnaire asked demographic questions (such as age, race, native, English speaker, gender, and socioeconomic background). In the second part, they completed the "Creative Achievement Questionnaire" which features 13 distinct areas of achievement (including: visual arts, music, dance, theater and film, architectural design, creative writing, humor, culinary arts, individual sports, team sports, entrepreneurship, scientific inquiry, and inventions).

The authors find that both greater diversity of experience and knowledge within a team and greater balance in individual contributions to group-level experiential diversity lead to increases in both the number of creative uses submitted and the number of valid uses submitted. On the contrary, diversity of gender, race, socioeconomic background, and personality do not have a measurable effect on creative production when accounting for diversity of experience.

\subsection{Cultural factors}

With the progressive shift of the globe's economic center of gravity toward Asia, and given the profound cultural gap between western and eastern societies, understanding culture impact on creative performances became crucial. A long-held stereotype affirms that Asian mainly excel in the logical and scientific domains, but they are, on average, weaker in the abstract tasks such as the ones requiring creativity. At this purpose we may cite the work by Morris and Leung [2010], who present a short literature review on the impact of the socio-cultural context on creativity when comparing two distant cultures. They provide as possible explanation of these differences the fact that social norms in the west encourage novelty and those in the east prioritize usefulness. Another viewpoint for these cultural differences is the one which was investigated by Saad et al. [2015]. They state a primary difference of western and Asian culture in their individualistic and collectivist orientation, respectively. In their experiment, implemented with a Canadian and a Taiwanese sample, they showed that the quantity of ideas generated was substantially higher for Canadians, whether the originality was higher for Taiwanese (contradicting the common claim of a lack of creativity by eastern). Moreover, Canadians were more confident in their creative abilities, and had a greater propensity to voice disagreement within group context.

The take-home message of the results of Saad et al. [2015] is essentially in line with the findings of Attanasi et al. [2019b], which, building on the same experimental setting of Attanasi et al. [2019a], investigate 
whether the impact on creativity may differ in diverse geographical locations. In this regard, they implemented the same experimental treatments described in Section 4.4 for Attanasi et al. [2019a] in two main cities of one western and one eastern country: Strasbourg, in France, and Ho Chi Minh city, in Vietnam. Experiments on creativity often include a post-experimental questionnaire investigating on additional characteristics of the subject pool (see Section 4.7 for a large discussion on the topic). The strength of this paper lies on pushing further the potential of this mixed-methods research. In order to deal with the culture effect on creativity, the authors made use of the results of the experiments, but also of the data collected through an extensive post-experimental questionnaire, investigating for social-identity effects. Indeed, participants answered a large set of questions, including items on their social and leisure habits, their tolerance and openness to different cultures, social capital, self-perceived creativity, self-perceived tolerance of the city they live in, etc. Matching the experimental results with the responses of the questionnaire, the authors found that differences between the two countries are well pronounced. In a western country such as France, social context has a strong influence on individual creativity. On the contrary, for Vietnam, as representative country for an eastern context, individual features such as intrinsic motivation or self-perceived creativity play a more important role on individual creativity. Moreover, in contrast with the stereotype we mentioned at the beginning of this section, and in line with Saad et al. [2015], no evidence of lower creative performance for easterners arises in Attanasi et al. [2019b].

\subsection{Non-monetary social incentives}

Even if mainly focused on the role of monetary rewards, a part of the experimental economic literature on creativity has given attention to some alternative forms of eliciting extrinsic motivation. In line with the psychological studies, rewards can be provided, for example, by the undirected effect of the pressure of performing in front of an audience, or in a coalition. Then, in this section, we analyze social incentives, interpreted a special type of non-monetary incentives.

In the third experiment of Ariely et al. [2009], the authors extend the focus of their analysis as compared to the first two parts of their paper, ${ }^{6}$ by investigating the role of social incentives, still in the context of a performance-based payment scheme. Formally, they examine the impact on performance when an audience watches a subject's work on a creativity task. In this specific experiment, they implemented a closed task which consisted in solving anagrams. Anagrams are jumble letters that can be made into one, and only one, very common word. The experiment was based on different trials, some trials being private, and some others

\footnotetext{
${ }^{6}$ The second part of the paper is not related to creativity. As such, it is out of the scope of our review.
} 
being public. In the private trials, all participants worked without being observed by anyone. In the public trials, one participant was chosen at random and asked to work in plain sight of the other participants, using a larger (and then, more visible) version of the same page that was used when anagrams were solved in private. The performance was evaluated in terms of the number of solved anagrams. The analysis revealed a chocking under pressure (namely, a crowding out effect of intrinsic motivation) by the social incentive: a large and significantly higher performance was found in the private condition.

In Charness and Grieco [2019], the authors introduce a follow-up experiment of the one we have already presented in Section 4.2 for Charness and Grieco [2013]. The same open-with-constraints and open tasks were implemented, but with the inclusion of an additional treatment within the flat payment scheme, that the authors call the "no ranking treatment". While in the "pure ranking treatment" participants were told that peers would have anonymously ranked the creativity of their work, in the new treatment no ranking occurred, maintaing the flat payment rule fixed. The authors find that the creativity scores were significantly higher when subjects were peer-ranked, for both open-with-constraints and open tasks. Simply being ranked (even anonymously) served as a form of non-monetary incentive that fostered creativity, no matter the degree of openness of the task. Therefore, here the main finding is that the specific social incentive has a positive effect on creativity.

\subsection{Personality traits}

As for the most recent literature in psychology, alsp in the experimental economic works personality traits are studied as complementary factors in the creative process. Then, papers in our review never investigate this topic as primary research question. Instead, they all complement their reflection with a final investigation about the correlation between the creative performances, and some personal characteristics of the subjects that were involved in the experiment. For this reason and following the same pattern, we decided to conclude our survey by making a point on this important aspect, following the categories provided in Table 5, where the same papers of Tables 2-4 are classified within each category of subjects' idiosyncratic, psychological and cognitive features.

At first, we underline how in experimental economics the subject pool is most of the times composed by undergraduate students. This is also true for our 13 works on creativity, in which we find only 4 examples where all or part of the experimental subjects are not students. ${ }^{7}$ Additionally, we categorize the personal

\footnotetext{
${ }^{7}$ The exceptions are: citizens from a rural village in India in Ariely et al. [2009] - Exp. 1; experts in creativity in Attanasi et al. [2019a] and Attanasi et al. [2019b], and elite software developers in Boudreau and Lakhani [2011]
} 
traits which are mostly investigated according to our review in the following four groups: demographic, cognitive style, risk and ambiguity attitudes.

Table 5: Subject pool and elicited personality traits

\begin{tabular}{|l|c|c|ccc|} 
& & & & \\
\hline
\end{tabular}

The demographic analysis includes the investigation of some idiosyncratic variables, such as age, sex, educational level, and origins. Regardless of the fact that it is pretty standard in experimental economics to include such controls on the subject pool, it is a common statement of the papers in our review that these characteristics have in general non-significant effects on the level of creativity. This statement holds with very few exceptions, such as for Charness and Grieco [2019], who discuss a marginally significant gender effect of a higher creative score for males. The same result may be observed in Bradler [2015]. Still Charness and Grieco [2019] find an interesting result consistent with the psychological research on the role of the characteristics of siblings: later-born subjects are generally less prone to conservatism and conformism, thus they turn to be more creative.

Cognitive style includes different variables, such as social habits, social capital, tolerance, open mindness, self-assessment of creativity, leisure habits, etc. We find some interesting hints on the role of these traits in the papers by Attanasi et al. [2019a,b]. As main results, Attanasi et al. [2019a] underline how "so- 
cial capital," i.e., subject's perception that in general most people can be trusted, turns out to have a positive effect on creativity. This is the consequence of a higher willingness to cooperate in group. On the contrary, "city tolerance," i.e., subject's perception to live in a tolerant city, has a negative correlation. This is the drawback effect of a wish to maintain a satisfying status quo, which then decreases the need for putting efforts in improving it. Attanasi et al. [2019b] remark the effects of the social habits and the leisure activities, that positively influence creativity in western participants, and the role of the intrinsic motivation and the self-perception of their own creativity, that enhance creativity of easterners.

The relation between risk attitude and creativity is standard since the earliest studies in psychology (see Section 2.1). Regardless the general lack of consensus, the predominant view of psychologists, which is also confirmed by the literature on economics of innovation, sees risk-lovers as more talented in creativity tasks. This statement, however, is not confirmed by our literature review in experimental economics. It is in fact partially validated only by Eckartz et al. [2012] and by Bradler [2015], that found that risk-loving people prefer choosing more risky payment schemes such as performance-based or tournament, instead of ensuring their payoff in a flat payment. However, the focus of their analysis on risk attitude is on how it correlates with the choice of the payment scheme, rather than on the effects on the creative performances. For the latter, Attanasi et al. [2019a,b] find a counterintuitive result, as risk aversion is shown to have a positive effect on a sub-sample of the subject pool, i.e., for undergraduate students in France (while no effect is found by considering the whole subject pool). Attanasi et al. [2019a] justify this finding providing different explanations, among which the most convincing relies on the features of their open-with-constraints creativity task: risk-averse subjects would be more accurate in satisfying the imposed constraints. A lack of effect of subjects' risk aversion over their assessed level of creativity is the conclusion of all the other works in Table 5 investigating this dimension.

The study of the relation between ambiguity attitude and creativity is instead less common in the reviewed experimental literature (we find it only in the works or Charness and Grieco [2013, 2019] and of Attanasi et al. [2019a,b]), maybe also because - differently from risk attitude - it is not an explored topic in either the psychological literature and in the literature on economics of innovation. ${ }^{8}$ Experimental results agree that a subject's aversion to ambiguity is not a relevant factor for his/her performance in a creativity task. In fact, Charness and Grieco [2019], find no correlation between ambiguity aversion and creativity in their open task. They find that ambiguity aversion has only a slightly significant negative effect on creativity

\footnotetext{
${ }^{8}$ For the different definitions of risk attitude (toward choice with known probabilities) and ambiguity attitude (toward choice with unknown probabilities), see, e.g., Attanasi and Montesano [2012].
} 
in their open-with-constraints task. In the same open-with-constraints task, Attanasi et al. [2019a,b] do not report any significant effect of ambiguity aversion on creativity for both experts and students (for the latter, both in France and in Vietnam). Considered together, all these results seem to show that the absence of any relation between ambiguity attitude and creativity generation holds independently of the type of creativity task.

\section{Conclusions}

Creativity has been shown to be important for economic development [Aghion and Howitt, 2008]. At macro level, it is a basic economic input that strongly shapes technological change [Mokyr, 1992]; at household level, fostering creativity may have the side effect of increasing well-being of individuals [Dolan and Metcalfe, 2012]. Finally, Romer [1993] has argued that the lack of creative ideas has played a role in condemning nations to poverty as in the case of lack of tangible assets. Even if most often referred to as novelty or economics of ideas, studies on creativity have gained their place in addressing the central economic question of why some economies thrive while others languish, transcending the walls of standard economics [see the works of the two Nobel Prize winners in 2006 and in 2018, respectively, Phelps, 2013, Romer, 1992].

The experimental research on creativity is the leitmotiv of our contribution. Throughout our survey, we try to stress how controlled laboratory experiments are the natural way to perform objective measurements of creativity and clean tests of the impact on creativity of individual and environmental factors. For this reason, we have put our focus on experimental economic studies of creativity, with a particular attention on the role of incentives. Even if "it may seem a presumptuous undertaking to inquire into the biological origins of mental creativity when we are still unable to define the chemistry of a simple muscle twitch" [citing the preface of Koestler, 1964], our review highlights how, despite less than fifteen years of production on the topic, experimental economics has already provided many variegate directions of research, shading light on some crucial points inherited from the psychological literature, and mediated by the management literature.

More precisely, we have identified and discussed six main contributions of experimental economic studies to the measurement of creativity and the understanding of its individual, social and organizational determinants. Namely, the impact on creativity of: (1) low vs high monetary incentives, (2) the interplay of monetary incentives and tasks, (3) within-group competition, (4) within-group cooperation, (5) cultural factors, and (6) non-monetary social incentives. Furthermore, and equally importantly, we have classified the studies on creativity that we have found in the experimental economics literature not only according to the 
aforementioned contributions, but also disentangling by the type of creativity task proposed to the subjects (e.g., verbal, mathematical, etc.) and whether it referred to close or open creativity (see Table 3), as well as by the way in which creativity was assessed (see Table 4). In the spirit of a meta-study, we have also considered the key features of standard experimental procedures in economics, and disentangled the studies we have found according to the type of subject pool and the elicited personality traits (see Table 5), the size and the currency of monetary incentives (see Table 2), the type of incentive scheme and whether it was implemented at an individual or group level (see Table 4). This "meta-study" has allowed us to show that, for the time being, the lack of a final say about the determinants of creativity in economics might be due to the fact that, given a similar research question - picked among the six we mention above -, there are not two fully comparable experimental studies implementing the same experimental conditions (i.e., comparable subject pool, type of creativity task, system of creativity assessment, size of monetary incentives, type of incentive scheme, etc.). Therefore, further experimental studies on creativity in economics are needed in order to produce a strand of literature that could provide robust findings.

We also notice that, besides a low absolute number of experimental studies on creativity in economics, there are important lines of research - which are trend topics in the general economic literature - that are fully unexplored from an experimental point of view. As leading example, we observe a fairly small interest of the experimental economic community in the issue of group creativity. In Section 2 we have shown that this is one of the pillars of the experimental research on creativity in both psychology and management. In contrast, few experiments of our review in Sections 3 and 4 have focused on the topic, and they have simply investigated some competition or cooperation effects of group tasks implementations. However, some longstanding organizational issues are totally neglected. Among others, we refer here to issues on inter-firm networks and the various mechanisms which underlie formation of ties between firms, and their performance effects in generating knowledge. Llerena and Ozman [2013], for example, highlight through an agent-based simulation the consequences of irreversibility in partner choice in inter-firm collaborations and the effects of the emerging network structures. An experimental validation of such network effects on the idea generation process represents a promising future line of research to give a response to the many questions about the behavioral implications of such theories.

Finally, as for the general economic literature on creativity, also in the community of experimentalists creativity is often considered at a "point in time," as a "moment of grace" providing us with the good brilliant idea. Many studies have questioned this simplified representation of creativity [see, e.g., Cohendet et al., 2020], by proposing, instead, a definition of creativity as a process with duration and phases [see Puccio 
and Grivas, 2009] and with different paths, where the final construct is strictly path-dependent [see, e.g., Perry-Smith and Mannucci, 2017]. A possible development of the experimental economic research is to analyze the interactions on creativity of a group over time, for example by modeling them via a dynamic network analysis.

\section{References}

P. Aghion and P. W. Howitt. The economics of growth. MIT press, 2008.

M. Agogué, A. Kazakçi, A. Hatchuel, P. Le Masson, B. Weil, N. Poirel, and M. Cassotti. The impact of type of examples on originality: Explaining fixation and stimulation effects. Journal of Creative Behavior, 48 (1):1-12, 2014a.

M. Agogué, N. Poirel, A. Pineau, O. Houdé, and M. Cassotti. The impact of age and training on creativity: A design-theory approach to study fixation effects. Thinking Skills and Creativity, 11:33-41, 2014b.

T.M. Amabile. Social psychology of creativity: A consensual assessment technique. Journal of Personality and Social Psychology, 43:997-1013, 1982a.

T.M. Amabile. Children's artistic creativity: Detrimental effects of competition in a field setting. Personality and Social Psychology Bulletin, 8:573-578, 1982b.

T.M. Amabile. The social psychology of creativity. New York: Springer-Verlag, 1983.

T.M. Amabile. Motivation and creativity: Effects of motivational orientation on creative writers. Journal of Personality and Social Psychology, 48:393-399, 1985.

T.M. Amabile and J. Pillemer. Perspectives on the social psychology of creativity. Journal of Creative Behavior, 46(1):3-15, 2012.

D. Ariely, U. Gneezy, G. Loewenstein, and N. Mazar. Large stakes and big mistakes. Review of Economic Studies, 76(2):451-469, 2009.

G. Attanasi and A. Montesano. The price for information about probabilities and its relation with risk and ambiguity. Theory and Decision, 73(1):125-160, 2012.

G. Attanasi, N. Georgantzís, V. Rotondi, and D. Vigani. Lottery-and survey-based risk attitudes linked through a multichoice elicitation task. Theory and Decision, 84(3):341-372, 2018. 
G. Attanasi, Y. Curci, P. Llerena, and G. Urso. Intrinsic vs. extrinsic motivators on creative collaboration: The effect of sharing rewards. Technical Report 2019-37, Bureau d'Economie Théorique et Appliquée, UDS, Strasbourg, 2019a.

G. Attanasi, Y. Curci, P. Llerena, A.C. Pinate, M.P. Ramos-Sosa, and G. Urso. Looking at creativity from east to west: Risk taking and intrinsic motivation in socially and culturally diverse countries. Technical Report 2019-38, Bureau d'Economie Théorique et Appliquée, UDS, Strasbourg, 2019b.

P. Azoulay, J.S.G Zivin, and G. Manso. Incentives and creativity: evidence from the academic life sciences. RAND Journal of Economics, 42(3):527-554, 2011.

M. Baer, G. R. Oldham, and A. Cummings. Rewarding creativity: when does it really matter? Leadership Quarterly, 14:569-586, 2003.

A. Bäker, W. Güth, K. Pull, M. Stadler, and F. Schiller. Creativity, analytical skills, personality traits, and innovation game behavior in the lab: An experiment. Technical Report 44, University of Tübingen Working Papers in Economics and Finance, 122011.

L. Baloche. Creativity and cooperation in the elementary music classroom. Journal of Creative Behavior, 28:255-265, 121994.

F. Barron. Creative vision and expression in writing and painting. In Conference on the creative person, Berkeley, CA: University of California, Institute of Personality Assessment and Research, 1961.

M.N. Bechtoldt, Choi H.S., and B.A. Nijstad. Individuals in mind, mates by heart: Individualistic selfconstrual and collective value orientation as predictors of group creativity. Journal of Experimental Social Psychology, 48:834-848, 2012.

R. Bénabou and J. Tirole. Intrinsic and extrinsic motivation. Review of Economic Studies, 70:489-520, 2003.

K.J. Boudreau and K.R. Lakhani. " fit" : Field experimental evidence on sorting, incentives and creative worker performance. Technical report, Harvard Business School, 2011.

R. Bouncken, A. Brem, and S. Kraus. Multi-cultural teams as sources for creativity and innovation: The role of cultural diversity on team performance. International Journal of Innovation Management, 20(1), 2015. 
C. Bradler. How creative are you? - an experimental study on self-selection in a competitive incentive scheme for creative performance. Technical Report 15-021, ZEW - Centre for European Economic Research, 2015.

C. Bradler, S. Neckermann, and A.J. Warnke. Incentivizing creativity: A large-scale experiment with tournaments and gifts. Technical Report 16-040, ZEW - Centre for European Economic Research, 2016.

T. Burger-Helmchen, C. Hussler, and P. Cohendet. Les grand auteurs en Management de l'Innovation et de la créativité. Editions EMS, 2016.

G. Charness and D. Grieco. Individual creativity, ex-ante goals and financial incentives. Technical report, UC Santa Barbara: Department of Economics., 2013.

G. Charness and D. Grieco. Creativity and financial incentives. Technical report, CESifo Area Conference on Behavioural Economics., 2014.

G. Charness and D. Grieco. Creativity and Incentives . Journal of the European Economic Association, 17 (2):454-496, 2019.

C.X. Chen, M.G. Williamson, and F.H. Zhou. Reward system design and group creativity: An experimental investigation. Accounting Review, 87(6):1885-1911, 2012.

R.Y.J. Chua, Y. Roth, and J. Lemoine. The impact of culture on creativity: How cultural tighness and cultural distance affect global innovation crowdsourcing work. Administrative Science Quarterly, 60(2): 189-227, 2015.

P. Cohendet, P. Llerena, , and L. Simon. The innovative firm: nexus of the communities and creativity. Revue d'Economie Industrielle, 7:129-130, 2010.

P. Cohendet, P. Llerena, , and L. Simon. The Routinization of Creativity. Lessons from the case of a Videogame Creative Powerhouse. Journal of Economics and Statistics, 234(2/3):120-141, 2014.

P. Cohendet, O. Dupouet, R. Naggar, and R. Rampa. Ideation processes and the dynamics of organizations. Technical report, Working Paper HEC Montréal - MosaiC, 2020.

M.A. Collins and T.M. Amabile. Motivation and creativity. In Robert J. Sternberg, editor, Handbook of Creativity, pages 297-312. Cambridge University Press, Cambridge, England, 1999. 
J. Condry. Enemies of exploration: Self-initiated versus other-initiated learning. Personality and Social Psychology, 35:459-477, 1977.

P. Crosetto and A. Filippin. A theoretical and experimental appraisal of four risk elicitation methods. Experimental Economics, 19(3):613-641, 2016.

J. Dandy, Brewer N., and R. Tottman. Self-consciousness and performance decrements within a sporting contextl. Journal of Social Psychology, 141:150-152, 2001.

R. De Fillippi, G. Grabher, and C. Jones. Introduction to paradoxes of creativity: managerial and organizational challenges in the cultural economy. Journal of Organizational Behavior, 28:511-521, 2007.

T. Dewett. Liking intrinsic motivation, risk taking, and employee creativity in an $\mathrm{r} \& \mathrm{~d}$ environment. $R \& D$ Management, 37(3):197-208, 2007.

P. Dolan and R. Metcalfe. Measuring subjective wellbeing: Recommendations on measures for use by national governments. Journal of Social Policy, 41:409-427, 2012.

E.G. Dutcher and C.S Rodet. Which two heads are better than one? uncovering the positive effects of diversity in creative teams. Technical Report 89982, MPRA Working Paper Series, 2018.

K. Eckartz, O. Kirchkamp, and D. Schunk. How do incentives affect creativity? Technical Report 4049, CESifo Working Paper Series, 2012.

R. Eisenberger and L. Rhoades. Incremental effects of reward on creativity. Journal of Personality and Social Psychology, 81:728-741, 2001.

R. Eisenberger, S. Armeli, and J. Pretz. Can the promise of reward increase creativity? Journal of Personality and Social Psychology, 74:704-714, 1998.

R. Eisenberger, F. Haskins, and P. Gambleton. Promised reward and creativity: Effects of prior experience. Journal of Experimental Social Psychology, 35:308-325, 1999.

S. Erat and U. Gneezy. Incentives for creativity. Experimental Economics, 19:269-280, 2016.

N. Erkal, L. Gangadharan, and B.H. Koh. Monetary and non-monetary incentives in real-effort tournaments. European Economic Review, 101:528-545, 2018.

H.J. Eysenck. Creativity and personality: Suggestions for a theory. Psychological Inquiry, 4:147-178, 1993. 
G. Feist. Influence of personality on artistic and scientific creativity. Handbook of Creativity, pages 273-296, 1999.

G. Feist. How development and personality influence scientific thought, interest, and achievement. Review of General Psychology, 10:163-182, 2006.

D. Galenson. A portrait of the artist as a young or old innovator: Measuring the careers of modern novelists. Historical Methods: A Journal of Quantitative and Interdisciplinary History, 39:51-72, 2004.

B. Gerhart and M. Fang. Pay, intrinsic motivation, extrinsic motivation, performance, and creativity in the workplace: Revisiting long-held beliefs. Annual Review of Organizational Psychology and Organizational Behavior, 2:489-521, 2015.

J.A. Glover. Risky shift and creativity. Social Behavior and Personality: An International Journal, 5: 317-320, 1977.

J.A. Glover and F. Sautter. An investigation of the relationship of four components of creativity to locus of control. Social Behavior and Personality: An International Journal, 4:257-260, 1976.

D.P. Gross. Creativity under fire: The effects of competition on creative production. Technical Report 25057, NBER Working Paper Series, 2018.

J.P. Guilford. Creativity. American Psychologist, 5:444-454, 1950.

J.P. Guilford. Creativity: A quarter century of progress. In I. A. Taylor and J. W. Getzels, editors, Perspectives in creativity, pages 37-59. Aldine, Chicago, 1975.

D. Hocevar. Intelligence, divergent thinking, and creativity. Intelligence, 4(1):25-40, 1980.

S.L. Hunsaker. Outcomes of creativity training programs. Gifted Child Quaterly, 49(4):292-299, 2005.

S.J. Kachelmeier, L.W. Wang, and M.G. Williamson. The role of incentives in sustaining high-creativity production. Technical report, 2015.

S.S. Kahai, J.J. Sosik, and B.J. Avolio. Effects of leadership style, anonymity, and rewards on creativityrelevant processes and outcomes in an electronic meeting system context. Leardership Quarterly, 14: 499-524, 2003.

A. Koestler. The act of creation. Macmillan, 1964. 
D.M. Kreps. Intrinsic motivation and extrinsic incentives. American Economic Review, 87(2):359-364, 1997.

K. Laske and M. Schröder. Quantity, quality and originality: The effects of incentives on creativity. Technical report, Beiträge zur Jahrestagung des Vereins für Socialpolitik, 2017.

P. Llerena and M. Ozman. Networks, irreversibility and knowledge creation. Journal of Evolutionary Economics, 23(2):431-453, 2013.

D.W. Mackinnon. Personality and the realization of creative potential. American Psychologist, 20:273-281, 1965.

S.A. Mednick. The associative basis of the creative process. Psychological Review, 69(3):220-232, 1962.

P.R. Merrifield, J.P. Guilford, P.R. Christensen, and J.W. Frick. Interrelationships between certain abilities and certain traits of motivation and temperament. Journal of General Psychology, 65:57-74, 1961.

A. Mohnen and A. Ostermaier. Incentives for creativity: Limits of objective performance evaluation. Technical report, TUM School of Management, 2013.

J. Mokyr. The level of riches: Technological creativity and economics progress. Oxford University Press, 1992.

M. Morris and K. Leung. Creativity east and west: Perspectives and parallels. Management and Organization Review, 6:313-327, 2010.

R. Ochse. Before the gates of excellence: The determinants of creative genius. Cambridge University Press, 1990.

A. Osborn. Applied imagination: Principles and procedures of creative thinking. New York: Scribner's, 1953.

J.E. Perry-Smith and P.V. Mannucci. From creativity to innovation: The social network drivers of the four phase of the idea journey. Academy of Management Review, 42(1):53-79, 2017.

E.S. Phelps. Mass flourishing: How grassroots innovation created jobs, challenge, and change. Princenton University Press, 2013. 
G. Puccio and C. Grivas. Examining the relationship between personality traits and creativity styles. Creativity and Innovation Management, 18(4):247-255, 2009.

P. Romer. Two strategies for economic development: Using ideas and producing ideas. World Bank Economic Review, 6(1):63-91, 1992.

P. Romer. Ideas gaps and object gaps in the economic development. Journal of Monetary Economics, 32 : 543-573, 1993.

G. Saad, M. Cleveland, and L. Ho. Individualism-collectivism and the quantity versus quality dimensions of individual and group creative performance. Journal of Business Research, 68:578-586, 2015.

E. Sanjiv and V. Krishnan. Managing delegated search over design spaces. Management Science, 58: 606-623, 2012.

D.K. Simonton. Sociocultural context of individual creativity: A trans-historical time-series analysis. Journal of Personality and Social Psychology, 32:1119-1133, 1975.

D.K. Simonton. Thematic fame and melodic originality in classical music: A multivariate computer-content analysis. Journal of Personality, 48:206-219, 1980.

D.K. Simonton. Origins of genius: Darwinian perspectives on creativity. New York: Oxford University Press, 1999.

B. Slavich and S. Svejenova. Managing creativity: A critical examination, synthesis, and new frontiers. European Management Review, 13:237-250, 2016.

S. Taggar. Group composition, creative synergy, and group performance. Journal of Creative Behavior, 35 (4):261-286, 2001.

S. Taggar. Individual creativity and group ability to utilize individual creative resources: A multilevel model. Academy of Management Journal, 45(2):315-330, 2002.

S. Taggar and M. Neubert. Impact of poor performers on team outcomes: An empirical examination of attribution theory. Personnel Psychology, 57:935-968, 2004.

D.W. Tegano, D.J. III Moran, and J.K. Sawyers. Creativity in early childhood classrooms. Washington, DC: National Education Association, 1991. 
E.P. Torrance. The torrance tests of creative thinking: Norms-technical manual. Lexington, MA: Personnel Press, 1966.

O. Toubia. Idea generation, creativity, and incentives. Marketing Science, 25(5):411-425, 2006.

V. Tyagi, Y. Hanoch, S.D. Hall, M. Runco, and S.L. Denham. The risky side of creativity: Domain specific risk taking in creative individuals. Frontiers in Psychology, 8(145), 2017.

L.C. Vincent and M. Kouchaki. Creative, rare, entitled, and dishonest: How commonality of creativity in one's group decrease an individual's entitlement and dishonesty. Academy of Management Journal, 59: 1451-1473, 2016.

S.D. Williams. Personality, attitude, and leader influences on divergent thinking and creativity in organizations. European Journal of Innovation Management, 7(3):187-204, 2004.

X. Zhang and K.M. Bartol. Liking empowering leadership and employee creativity: The influence of psychological empowerment, intrinsic motivation, and creative process engagement. Academy of Management Journal, 53(1):107-128, 2010. 